\title{
CONTROLLED APPROXIMATION AND A CHARACTERIZATION OF THE LOCAL APPROXIMATION ORDER ${ }^{1}$
}

\author{
C. DE BOOR AND R.-Q. JIA
}

\begin{abstract}
The local approximation order from a scale $\left(S_{h}\right)$ of approximating functions on $\mathbf{R}^{m}$ is characterized in terms of the linear span (and its Fourier transform) of the finitely many compactly supported functions $\varphi$ whose integer translates $\varphi(\cdot-j), j \in Z^{m}$, span the space $S=S_{1}$ from which the scale is derived. This provides a correction of similar results stated and proved, in part, by Strang and Fix.
\end{abstract}

The term "controlled approximation" was introduced in 1970 by Strang [St]; see also [FS]. It concerns approximations of the form $\sum_{\varphi \in \Phi} \varphi * c_{\varphi}$, with $\Phi$ a finite collection of functions on $\mathbf{R}^{m}$ of compact support and $\varphi * c$ the function obtained from $\varphi$ by convolution with some "sequence" $c: \mathbf{Z}^{m} \rightarrow \mathbf{R}$; i.e.,

$$
\varphi * c:=\sum_{j \in \mathbf{Z}^{m}} \varphi(\cdot-j) c(j) \text {. }
$$

If, more generally, $c$ is some function on $\mathbf{R}^{m}$, we will still just write $\varphi * c$ instead of the correct but more complicated $\varphi *\left(\left.c\right|_{\mathbf{Z}^{m}}\right)$.

The function $u$ to be approximated lies in the Sobolev space $W_{p}^{k}\left(\mathbf{R}^{m}\right)$ with norm

$$
\|u\|_{k, p}:=\sum_{j \leqslant k}|u|_{j, p}
$$

where

$$
|u|_{j, p}:=\sum_{|\alpha|=j}\left\|D^{\alpha} u\right\|_{p}
$$

and

$$
\|u\|_{p}:=\|u\|_{L_{p}\left(\mathbf{R}^{m}\right)}
$$

We denote by $W_{p, c}^{k}\left(\mathbf{R}^{m}\right)$ the subspace of $W_{p}^{k}\left(\mathbf{R}^{m}\right)$ of compactly supported functions.

The approximations are, more explictly, of the form

$$
\sigma_{h}\left(\sum_{\varphi \in \Phi} \varphi * c_{\varphi}^{h}\right) / h^{m / p} \text { with } \sigma_{h} f:=f(\cdot / h) .
$$

Received by the editors November 19, 1984.

1980 Mathematics Subject Classification. Primary 41A25, 41A15, 41A63, 65N30.

Key words and phrases. Controlled approximation, approximation order, multivariate, box splines, finite element analysis, Fourier series.

${ }^{1}$ Sponsored by the United States Army under Contract No. DAAG29-80-0041. 
Concerning the degree of approximation to $u \in W_{p}^{k}\left(\mathbf{R}^{m}\right)$ achievable by proper choice of the weights $c_{\varphi}^{h}$, Strang and Fix [SF, Theorem II] state the following result. In its statement and subsequent analysis, the normalized multivariate monomials appear often enough to deserve an abbreviation of their own. We will use [ $]^{\alpha}$ to stand for the function $\mathbf{R}^{m} \rightarrow \mathbf{R}: x \rightarrow x^{\alpha} / \alpha$ ! (and will use standard multi-index notation throughout). In particular, $D^{\beta}[]^{\alpha}=[]^{\alpha-\beta}$, and this holds even when $\beta \nless \alpha$, since then [ $]^{\alpha-\beta}=0$, by convention. Further, $\pi_{j}$ will denote the collection of polynomials on $\mathbf{R}^{m}$ of total degree $\leqslant j$. Finally, $\hat{f}$ will denote the Fourier transform of $f$-i.e., $\hat{f}(\xi):=\int_{\mathbf{R}^{m}} e^{-i \xi x} f(x) d x$, with $\xi x$ the scalar product.

THEOREM SF. Let $\Phi$ be a finite subset of $W_{2, c}^{k-1}\left(\mathbf{R}^{m}\right)$. Then the following are equivalent:

(i) There exists a sequence $\left(\psi_{\alpha}\right)_{|\alpha|<k}$ in $\operatorname{span} \Phi$ which satisfies

(ia) $\hat{\psi}_{0}(0)=1, \hat{\psi}_{0}=0$ on $2 \pi \mathbf{Z}^{m} \backslash 0$;

(ib) $\sum_{\beta \leqslant \alpha}[-i D]^{\beta} \hat{\psi}_{\alpha-\beta}=0$ on $2 \pi \mathbf{Z}^{m}$ for $0<|\alpha|<k$.

(ii) There exists a sequence $\left(\psi_{\alpha}\right)_{|\alpha|<k}$ in $\operatorname{span} \Phi$ which satisfies

$$
[]^{\alpha}=\sum_{\beta \leqslant \alpha} \psi_{\alpha-\beta} *[]^{\beta} \text { for }|\alpha|<k .
$$

(iii) There exist some finitely supported $c_{\varphi}$ so that $\psi:=\sum_{\varphi \in \Phi} \varphi * c_{\varphi}$ satisfies $\hat{\psi}(0) \neq 0$, but $D^{\alpha} \hat{\psi}=0$ on $2 \pi \mathbf{Z}^{m} \backslash 0$ for $|\alpha|<k$.

(iv) For each $u \in W_{2}^{k}\left(\mathbf{R}^{m}\right)$ there exist weights $c_{\varphi}^{h}$ so that

(iva) $\left\|u-\sigma_{h}\left(\sum_{\varphi} \varphi * c_{\varphi}^{h}\right) / h^{m / 2}\right\|_{s, 2} \leqslant$ const $_{s} h^{k-s}|u|_{k, 2}, s=0, \ldots, k-1$;

(ivb) $\Sigma_{\varphi}\left\|c_{\varphi}^{h}\right\|_{2}^{2} \leqslant$ const $\|u\|_{2}^{2}$.

For the very special case when $m=\# \Phi=1$, such results can already be found in [Sc]. In [SF] the special case when $\Phi$ consists of just one function (but $m$ is arbitrary) is treated first (see [SF, Theorem I]) and completely. However, for the general case, [SF] only gives a proof for the implications (i) $\Rightarrow$ (iii) $\Rightarrow$ (iv). In particular, the validity of (iv) $\Rightarrow$ (i) has recently been questioned. This was finally settled by Jia [J], who shows by a counterexample that (iv) does not imply (i) in general.

This raises the question of how to modify (iv) to obtain something equivalent to (i). This is a matter of changing the control over the form of the approximation as expressed by (ivb). In this connection it is very useful to recall that Dahmen and Micchelli [DM] quote Theorem SF in a modified form. The modification of importance here occurs in condition (iv), which they require to hold locally, as follows:

(iv)' For each $u \in W_{p}^{k}\left(\mathbf{R}^{m}\right)$ there exist weights $c_{\varphi}^{h}$ so that, for any closed domain $G \subset \mathbf{R}^{m}$,

(iva)'

$$
\left\|u-\sigma_{h} \sum_{\varphi} \varphi * c_{\varphi}^{h} h^{m / p}\right\|_{p}(G) \leqslant \text { const } h^{k}|u|_{k, p}\left(B_{r h}(G)\right),
$$

(ivb)

$$
\left\{\sum_{G \cap \operatorname{supp} \varphi(\cdot / h-j) \neq \varnothing}\left|c_{\varphi}^{h}(j)\right|^{p}\right\}^{1 / p} \leqslant \text { const }\|u\|_{p}\left(B_{r h}(G)\right)
$$

holds for some const and $r$ independent of $h, G, u$. 
Here, $B_{d}(G):=\left\{x \in \mathbf{R}^{m}: \operatorname{dist}(x, G)<d\right\}$.

If (iv)' holds, then, with a reference to [St], [DM] say that $\Phi$ provides "controlled $L_{p}$-approximation of order $k$ '. They do not comment on the fact that (iv)' is a strengthening of (iv), and they refer to [SF] for a proof of the implication (iv) ${ }^{\prime} \Rightarrow$ (i).

As it turns out, (iv)' does indeed imply (i). But it is the localness rather than the control that does the job. For this reason we propose here to abandon the notion of "controlled approximation order" in favor of "local approximation order". We say that $\Phi$ provides "local $L_{p}$-approximation of order $k$ " in case the following condition holds:

(iv)" For each $u \in W_{p}^{k}\left(\mathbf{R}^{m}\right)$ there exist weights $c_{\varphi}^{h}$ so that (iva)"

$$
\left\|u-\sigma_{h} \sum_{\varphi} \varphi * c_{\varphi}^{h}\right\|_{p} \leqslant \text { const } h^{k}|u|_{k, p}
$$

(ivb) $)^{\prime \prime}$

$$
c_{\varphi}^{h}(j)=0 \text { whenever } \operatorname{dist}(j h, \operatorname{supp} u)>r
$$

holds for some const and some $r$ independent of $h$ and $u$.

It is clear that (ivb)' is stronger than both (ivb) and (ivb)", but (ivb) and (ivb)" are not comparable. As we are about to show, (iv)" is the right modification of (iv) to give equivalence with (i). This shows that also (iv) ${ }^{\prime} \Rightarrow$ (i) and so validates the version of Theorem SF in [DM].

THEOREM. Let $\Phi$ be a finite subset of $W_{p, c}^{0}\left(\mathbf{R}^{m}\right)$. Then the following statements are equivalent:

$\left(1^{\circ}\right)$ There exists a sequence $\left(\psi_{\alpha}\right)_{|\alpha|<k}$ in $\operatorname{span} \Phi$ which satisfies

$\left(1^{\circ} \mathrm{a}\right) \hat{\psi}_{0}(0)=1, \hat{\psi}_{0}=0$ on $2 \pi \mathbf{Z}^{m} \backslash 0$;

$\left(1^{\circ}\right.$ b) $\sum_{\beta \leqslant \alpha}[-i D]^{\beta} \hat{\psi}_{\alpha-\beta}=0$ on $2 \pi \mathbf{Z}^{m} \backslash 0$ for $0<|\alpha|<k$.

$\left(2^{\circ}\right)$ There exists a sequence $\left(\psi_{\alpha}\right)_{|\alpha|<k}$ in $\operatorname{span} \Phi$ such that

$$
[]^{\alpha}-\sum_{\beta \leqslant \alpha} \psi_{\alpha-\beta} *[]^{\beta} \in \pi_{|\alpha|-1} \text { for }|\alpha|<k .
$$

$\left(3^{\circ}\right)$ There exist some finitely supported $c_{\varphi}$ so that $\psi:=\sum_{\varphi \in \Phi} \varphi * c_{\varphi}$ satisfies

$$
[]^{\alpha}-\psi *[]^{\alpha} \in \pi_{|\alpha|-1} \text { for }|\alpha|<k \text {. }
$$

$\left(4^{\circ}\right)$ For all $p \in[1, \infty], \Phi$ provides local $L_{p}$-approximation order $k$.

$\left(5^{\circ}\right)$ For some $p \in[1, \infty]$, $\Phi$ provides local $L_{p}$-approximation order $k$.

REMARKS. Condition $\left(1^{\circ}\right)$ differs from (i) in that the latter requires, additionally, that $\sum_{\beta \leqslant \alpha}[-i D]^{\beta} \hat{\psi}_{\alpha-\beta}=0$ at 0 . Already Dahmen and Micchelli [DM] prove that it is possible to get away with the weaker condition $\left(1^{\circ}\right)$.

Condition $\left(2^{\circ}\right)$ is, off-hand, weaker than (ii), but an inductive argument leads from $\left(2^{\circ}\right)$ to (ii).

Condition $\left(3^{\circ}\right)$ seems more useful to us in applications than the (equivalent) condition (iii). We note that induction gives the seemingly stronger statement 
$\left(3^{\circ}\right)^{\prime}$ There exist some finitely supported $c_{\varphi}$ so that $\psi:=\sum_{\varphi \in \Phi} \varphi * c_{\varphi}$ satisfies $\psi *[]^{\alpha}=[]^{\alpha}$ for $|\alpha|<k$.

The asserted equivalence between $\left(4^{\circ}\right)$ and $\left(5^{\circ}\right)$ shows that one might as well drop the qualifier " $L_{p}$-" and just speak of the local approximation order provided by $\Phi$.

Proof of THE THEOREM. While the main point of this note is the implication $\left(5^{\circ}\right) \Rightarrow\left(1^{\circ}\right)$, we give a proof of the entire implication cycle $\left(1^{\circ}\right) \Rightarrow\left(2^{\circ}\right) \Rightarrow \cdots \Rightarrow$ $\left(1^{\circ}\right)$. The arguments for $\left(1^{\circ}\right) \Rightarrow\left(2^{\circ}\right) \Rightarrow\left(3^{\circ}\right)$ are adaptations of those in [SF].

$\left(1^{\circ}\right) \Rightarrow\left(2^{\circ}\right)$. Observe that the Fourier transform of $\psi(x-\cdot)[]^{\beta}$ is $[i D]^{\beta}\left(e^{-i(\cdot) x} \hat{\psi}(-\cdot)\right)$. Hence, by Poisson's summation formula, we have

$$
\begin{aligned}
\psi *[]^{\beta}(x) & =\left.\sum_{j}[i D]^{\beta}\left(e^{-i \xi x} \hat{\psi}(-\xi)\right)\right|_{\xi=2 \pi j} \\
& =\sum_{j} \sum_{\gamma \leqslant \beta}[x]^{\gamma} e^{-2 \pi i j x}[-i D]^{\beta-\gamma} \hat{\psi}(-2 \pi j) .
\end{aligned}
$$

It follows that

$$
\begin{aligned}
\sum_{\beta \leqslant \alpha} \psi_{\alpha-\beta} *[]^{\beta} & =\sum_{j} \sum_{\beta \leqslant \alpha} \sum_{\gamma \leqslant \beta}[]^{\gamma} e^{-2 \pi i j()}[-i D]^{\beta-\gamma} \hat{\psi}_{\alpha-\beta}(-2 \pi j) \\
& =\sum_{j} e^{-2 \pi i j()} \sum_{\gamma \leqslant \alpha}[]^{\gamma} \sum_{\beta-\gamma \leqslant \alpha-\gamma}[-i D]^{\beta-\gamma} \hat{\psi}_{(\alpha-\gamma)-(\beta-\gamma)}(-2 \pi j) \\
& =[]^{\alpha}+\sum_{\gamma<\alpha}[]^{\gamma} \sum_{\delta \leqslant \alpha-\gamma}[-i D]^{\delta} \hat{\psi}_{\alpha-\gamma-\delta}(0)
\end{aligned}
$$

the last equation by $\left(1^{\circ}\right)$.

$\left(2^{\circ}\right) \Rightarrow\left(3^{\circ}\right)$. With $\gamma:=(k, \ldots, k)$, the monomial [ $]^{\gamma}$ and its integer shifts span the space $\pi_{k}(\mathbf{R}) \times \cdots \times \pi_{k}(\mathbf{R})$. This implies that there exist finitely supported sequences $c_{\beta}$ so that

$$
[]^{\gamma} * c_{\beta}=[]^{\gamma-\beta} \text { for } \beta \leqslant \gamma
$$

On applying $D^{\gamma-\alpha}$ to both sides, we find that []$^{\alpha} * c_{\beta}=[]^{\alpha-\beta}$; hence,

$$
c_{\beta} *[]^{\alpha}=[]^{\alpha-\beta} \text { on } \mathbf{Z}^{m} \quad \text { for } \alpha, \beta \leqslant \gamma .
$$

Now set

$$
\psi:=\sum_{|\beta|<k} \psi_{\beta} * c_{\beta}
$$

Then

$$
\psi *[]^{\alpha}=\sum_{|\beta|<k} \psi_{\beta} * c_{\beta} *[]^{\alpha}=\sum_{|\beta|<k} \psi_{\beta} *[]^{\alpha-\beta}=\sum_{\beta \leqslant \alpha} \psi_{\beta} *[]^{\alpha-\beta} \in[]^{\alpha}+\pi_{|\alpha|-1},
$$

the inclusion by $\left(2^{\circ}\right)$.

The proof of $\left(3^{\circ}\right) \Rightarrow\left(4^{\circ}\right)$ follows the argument for [ $\mathrm{BH}$, Corollary to Theorem 6].

This leaves $\left(5^{\circ}\right) \Rightarrow\left(1^{\circ}\right)$. We approximate a tensor product of univariate B-splines - namely, the function

$$
u(x):=\prod_{\nu=1}^{m} M_{k+1}(x(\nu))
$$


with

$$
M_{k+1}(t):=\left[-\frac{k+1}{2},-\frac{k-1}{2}, \ldots, \frac{k+1}{2}\right](\cdot-t)_{+}^{k}, \quad t \in \mathbf{R}
$$

(see [Sc]). Since $u \in W_{p}^{k}\left(\mathbf{R}^{m}\right)$ for any $p \in[1, \infty]$, we can find weights $c_{\varphi}^{h}$ so that (iva)" and (ivb)" hold (whatever the $p$ might be). Set

$$
u_{h}:=\sigma_{h}\left(\sum_{\varphi} \varphi * c_{\varphi}^{h}\right)
$$

and consider the Fourier-Laplace transform

$$
\hat{f}(z):=\int e^{-i z x} f(x) d x, \quad z \in \mathbf{C}^{m},
$$

of the error $f:=u-u_{h}$. Since $u$ has compact support, so does $u_{h}$ by (ivb) ${ }^{\prime \prime}$; hence, so does $f$ uniformly in $h$. This means that supp $f$ lies in some ball $B_{a}$ of finite radius $a$ independently of $h$. Consequently,

and, by (iva)",

$$
|\hat{f}(z)|=\left|\int_{B_{a}} e^{-i z x} f(x) d x\right| \leqslant e^{a\|\operatorname{Im} z\|} \text { const }_{a}\|f\|_{p},
$$

$$
\|f\|_{p} \leqslant \text { const } h^{k}|u|_{k, p}
$$

This implies that

$$
|\hat{f}(z)| \leqslant \text { const } h^{k} \quad \text { for }\|\operatorname{Im} z\| \leqslant \text { const. }
$$

We can therefore invoke Cauchy's formula (see, e.g., $[\mathbf{R}])$ to get the estimate

$$
\left\|D^{\alpha} \hat{f}\right\|_{\infty}(\mathbf{R}) \leqslant \text { const } h^{k}
$$

The Fourier transform of $u$ is well known (see [Sc]); it is

$$
\hat{u}(z)=\prod_{\nu=1}^{m} C(z(\nu))^{k+1}
$$

where $C(t):=(\sin t / 2) /(t / 2)$ is Whittaker's cardinal function. From this we deduce that

$$
\hat{u}(0)=1
$$

and

$$
\lim _{h \rightarrow 0} \frac{\left(D^{\alpha} \hat{u}\right)(x / h)}{h^{k}}=0 \quad \text { for } x \in \mathbf{R}^{m} \backslash 0 \text { and }|\alpha|<k ;
$$

hence, by (1), $\hat{u}_{h}$ must satisfy corresponding conditions.

We now compute $\hat{u}_{h}$. Since

we find that

$$
\int e^{-i z x} \varphi(x / h-j) d x=h^{m} \hat{\varphi}(h z) e^{-i h z j},
$$

with

$$
\hat{u}_{h}(z)=\sum_{\varphi} \hat{\varphi}(h z) v_{\varphi, 0}^{h}(z)
$$

$$
v_{\varphi, 0}^{h}(z):=h^{m} \sum_{j} c_{\varphi}^{h}(j) e^{-i h z j}
$$


Thus, from (1) and (2),

$$
\lim _{h \rightarrow 0} \sum_{\varphi} \hat{\varphi}(0) v_{\varphi, 0}^{h}(0)=1
$$

Further,

$$
[D]^{\alpha}\left(\hat{\varphi}(h z) v_{\varphi, 0}^{h}(z)\right)=\sum_{\beta \leqslant \alpha} h^{|\beta|}[D]^{\beta} \hat{\varphi}(h z)(-i h)^{|\alpha-\beta|} v_{\varphi, \alpha-\beta}^{h}(z)
$$

with

$$
v_{\varphi, \gamma}^{h}(z):=h^{m} \sum_{j} c_{\varphi}^{h}(j)[j]^{\gamma} e^{-i h z j}
$$

each a $2 \pi / h$-periodic function. (Note that, for $\gamma=0$, this agrees with the earlier definition, as it should.) This implies that

$$
v_{\varphi, \gamma}^{h}(2 \pi j / h)=v_{\varphi, \gamma}^{h}(0),
$$

and therefore (1) and (3) give

$$
\sum_{\varphi} \sum_{\beta \leqslant \alpha} h^{|\alpha|}(-i)^{|\alpha-\beta|}[D]^{\beta} \hat{\varphi}(2 \pi j) v_{\varphi, \alpha-\beta}^{h}(0)=0\left(h^{k}\right)
$$

hence, for $|\alpha|<k$,

$$
\lim _{h \rightarrow 0} \sum_{\varphi} \sum_{\beta \leqslant \alpha}[-i D]^{\beta} \hat{\varphi}(2 \pi j) v_{\varphi, \alpha-\beta}^{h}(0)=0 \quad \text { for } j \in \mathbf{Z}^{m} \backslash 0 .
$$

From (4) and (5) we deduce $\left(1^{\circ}\right)$ as follows. By (4) we cannot have $\hat{\varphi}(0)=0$ for every $\varphi \in \Phi$. Without loss of generality we can therefore assume that, for some $\chi \in \Phi, \hat{\chi}(0)=1$, and $\hat{\varphi}(0)=0$ for all $\varphi \in \Phi \backslash \chi$. In particular, this implies with (4) that $\lim _{h \rightarrow 0} v_{\chi, 0}^{h}(0)=1$. Now consider the space $S$ of all vectors $w=\left(w_{\varphi, \gamma}\right)$ for which

$$
\lim _{h \rightarrow 0} \sum_{\varphi} \sum_{|\gamma|<k} w_{\varphi, \gamma} v_{\varphi, \gamma}^{h}(0)=0 .
$$

We claim that $S \perp$ contains a vector $w^{\prime}$ with $w_{\chi, 0}^{\prime}=1$. Indeed, if $w_{x, 0}^{\prime}=0$ for all $w^{\prime} \in S \perp$, then $(S \perp) \perp=S$ would contain the unit vector $\left(\delta_{\varphi \chi} \delta_{\gamma 0}\right)$; hence, $\lim _{h \rightarrow 0} v_{\chi, 0}^{h}(0)=0$ would follow.

With this, define

$$
\psi_{\gamma}:=\sum_{\varphi} w_{\varphi, \gamma}^{\prime} \varphi
$$

Then

$$
\hat{\psi}_{0}(0)=\sum_{\varphi} w_{\varphi, 0}^{\prime} \hat{\varphi}(0)=w_{\chi, 0}^{\prime} \hat{\chi}(0)=1
$$

Further, by (5), the vector $\left([-i D]^{\alpha-\gamma} \hat{\varphi}(2 \pi j)\right)_{\varphi, \gamma}$ is in $S$ for $|\alpha|<k$ and $j \in \mathbf{Z}^{m} \backslash 0$; therefore

$$
\sum_{\varphi} \sum_{\beta \leqslant \alpha} w_{\varphi, \alpha-\beta}^{\prime}[-i D]^{\beta} \hat{\varphi}(2 \pi j)=0 \quad \text { for }|\alpha|<k \text { and } j \in \mathbf{Z}^{m} \backslash 0,
$$


and this implies that

$$
\sum_{\beta \leqslant \alpha}[-i D]^{\beta} \hat{\psi}_{\alpha-\beta}=0 \text { on } 2 \pi \mathbf{Z}^{m} \backslash 0 \text { for }|\alpha|<k,
$$

as we wanted to show.

REMARK. In contrast to [SF], we assume $\Phi$ only to lie in $L_{p}$. For this reason we do not get (iva); i.e., we do not get simultaneous approximation to derivatives. But this is easily obtained under the assumption that $\Phi$ lie in an appropriately smoother space, using the quasi-interpolant constructed in the proof of $\left(3^{\circ}\right) \Rightarrow\left(4^{\circ}\right)$. In this connection we note that, using $\left(3^{\circ}\right)$ in the equivalent formulation $\left(3^{\circ}\right)^{\prime}$, such a quasi-interpolant for $u$ takes the particularly simple form

$$
\psi * u
$$

\section{REFERENCES}

[BH] C. de Boor and K. Höllig, B-splines from parallelepipeds, J. Analyse Math. 42 (1982/83), 99-115.

[DM] W. Dahmen and C. A. Micchelli, On the approximation order from certain multivariate spline spaces, J. Austral. Math. Soc. Ser B 26 (1984), 233-246.

[FS] G. Fix and G. Strang, Fourier analysis of the finite element method in Ritz-Galerkin theory, Stud. Appl. Math. 48 (1969), 265-273.

[J] R.-q. Jia, A counterexample to a result of Strang and Fix concerning controlled approximation, MRC TSR \# 2743, 1984.

[R] W. Rudin, Function theory in the unit ball of $C^{n}$, Grundlehren Math. Wiss., Vol. 241, Springer-Verlag, New York, 1980.

[Sc] I. J. Schoenberg, Contributions to the problem of approximation of equidistant data by analytic functions. A, B, Quart. Appl. Math. 4 (1946), 45-99, 112-141.

[St] G. Strang, The finite element method and approximation theory, Numerical Solution of Partial Differential Equations. II, SYNSPADE 1970 (B. Hubbard, ed.). Univ. of Maryland, College Park, 1971. pp. 547-583.

[SF] G. Strang and G. Fix, A Fourier analysis of the finite element variational method. Constructive Aspects of Functional Analysis (G. Geymonat, ed.), C.I.M.E., 1973, pp. 793-840.

Mathematics Research Center, 610 Walnut Street, Madison, Wisconsin 53705

Department of Mathematics, Zhejiang University, Hangzhou, Zhejiang, The People's RepubLIC OF CHINA 